\section{Beyond the Hype - Is 2-Photon Microscopy Right for You?}

\author{
Jason Kirk \\ University of Connecticut Health Center \\ jkirk@neuron.uchc.edu
}

Confocal microscopes have come a long way in the past decade. Not only are they more stable and easier to use than ever before, but their cost has dropped enough that multi-user facilities are finding competition from individual labs using the new breed of "personal" confocals. In fact it has, in some cases, become the de facto standard for fluorescence imaging regardless of whether the user actually has requirements for it or not.

But, researchers always have an ear out for something better. Enter 2-photon microscopy (2PLSM). The "bigger \& badder" cousin of the confocal microscope has become a new weapon in the arsenal of a microscopy industry that caters to researchers who can't wait to fill their labs with the latest and greatest imaging systems. Advertised by the industry and researchers alike as a technique that seems to solve most of the problems that plague confocal, "2-photon" has become the new buzzword in the vocabulary of researchers eager to enhance their fluorescence applications.

Looking beyond the hype typically associated with high-end imaging, and attempting to distinguish potential benefits from drawbacks, comes down to understanding why 2PLSM is right (or wrong) for your application. But how do you know if your application could really benefit from 2PLSM? And is it worth the effort?

The unique ability of a fluorochrome to absorb two photons of pulsed infrared light in the same quantum event, resulting in visible wavelength emission, has cleared a path for 2PLSM into mainstream imaging. This event occurs exclusively at the focal point of the objective lens yielding diffraction-limited excitation controlled entirely by the NA of the objective used. With no outof-plane excitation to block, exciting in 2-photon space allows for direct detection of emission without the need for light-robbing pinholes. This effect maintains (or in most cases enhances) threedimensional information while improving the signal-to-noise ratio of an image. This intrinsic three-dimensionality allows for much more precise 3D imaging compared to confocal.

With the aid of longer working distance objectives, a 2PLSM can scan much deeper into samples than confocal. Aberrations enhanced by convolutional effects from out-of-plane excitation in 1-photon space drastically reduce image quality in depths beyond 50 microns, whereas 2PLSM has no problem imaging through 200 microns or more without the signal degradation and resolution loss associated with confocal. In addition, photo damage from bleaching is virtually non-existent in out-of-focus planes, which opens up new doors in time course and 3D imaging with live cells.

One of the more high profile enhancements 2PLSM has brought is the ability to manipulate excitation wavelengths to the nanometer. This capability casts off the restrictions imposed by confocal manufacturers who only support a handful of laser wavelengths, and gives new life to dyes like fluorescent proteins BFP, CFP, YFP and other dyes close to/in the UV without the dramatic lens aberrations caused by confocal excitation in the UV.

While not having to worry about blasting samples with a laser in order to squeeze enough light through a pinhole to get a decent signal-to-noise ratio seems almost utopian, 2PLSM systems are exponentially more complex to use and maintain. There are some simple questions you can ask yourself when wondering if your application is right for 2PLSM. Does your application require:

1. Precise three-dimensional imaging in reasonably thick specimens?

2. Fluorescence excitation in extremely thick cross-sections?

3. Fluorochrome(s) close to/in the UV, or requires visible laser excitation not specifically available on commercial confocals?

4. Use of fluorochromes with a low tolerance for photo bleaching?

The four criterion expressed above are by no means absolute, however, they are the areas where 2PLSM excels and could enhance your imaging results compared to confocal. While 2PLSM does solve many of the headaches associated with confocal, it introduces many of its own that make most researchers wonder if spending over half a million dollars is a worthwhile move.

First, a multi-photon setup is large. It requires a dedicated room that is both strictly temperature and light controlled. Pulsed IR lasers have extremely long beam paths that even when uniquely folded create a housing over 4 feet long. Direct couple that to a scan head and microscope and you are looking at an isolation table at least 4 feet by 6 feet. You must also leave walking room around the ENTIRE table, as you need to have access to the laser and microscope workstation, which in most cases end up on opposite sides of the table.

By far the most complicated issue surrounding 2PLSM is the use of an ultrafast laser. While some laser manufacturers are introducing "hands-off" tunable IR lasers specifically designed for microscopy, the current crop of high-end lasers require users to have a fluent understanding of optics and the specific laser's requirements in order to properly adjust and maintain such a complex optical path. They require precise adjustments at as many as 5 or more locations throughout the cavity, while simultaneously monitoring 3 or more beam measurement variables. Additional variables such as room temperature, circulation of air/dust, and vibration isolation also have dramatic effects on their performance.

While these lasers are reasonably stable given proper precautions, biologists tend to change wavelengths often, which poses the greatest risk for user misalignment, as the beam must be optimized after every wavelength change. The popular use of spectral devices like a REES* can simplify wavelength changes by picking off part of the beam at output and displaying its spectral readout, but these and other spectrometers give you little/no information on beam alignment and proper mode-locking. Many other alignment issues also affect the delivery of the beam to the scan head. The relay mirrors transporting the beam to the microscope will only magnify slight misalignments at the measured output of the IR laser.

High-end IR lasers are often coupled to large pump sources, typically between 5 and 10 Watts. This can generate over a Watt of power (tuned to $\sim 800 \mathrm{~nm}$ ) upon output, which makes using these lasers inherently dangerous. Institutions may stipulate that the beam be interlocked at points of room entry, and users provide adequate notification of activity and post visible warning of its dangers. All of which I should mention are NOT automatically provided by laser manufacturers. While cases enclosing an IR cavity restrict output, anyone who has used an IR laser knows you often have the cover away from the cavity for access to the beampath. 
E X C E L LE N C E ... M A G N I F I E D

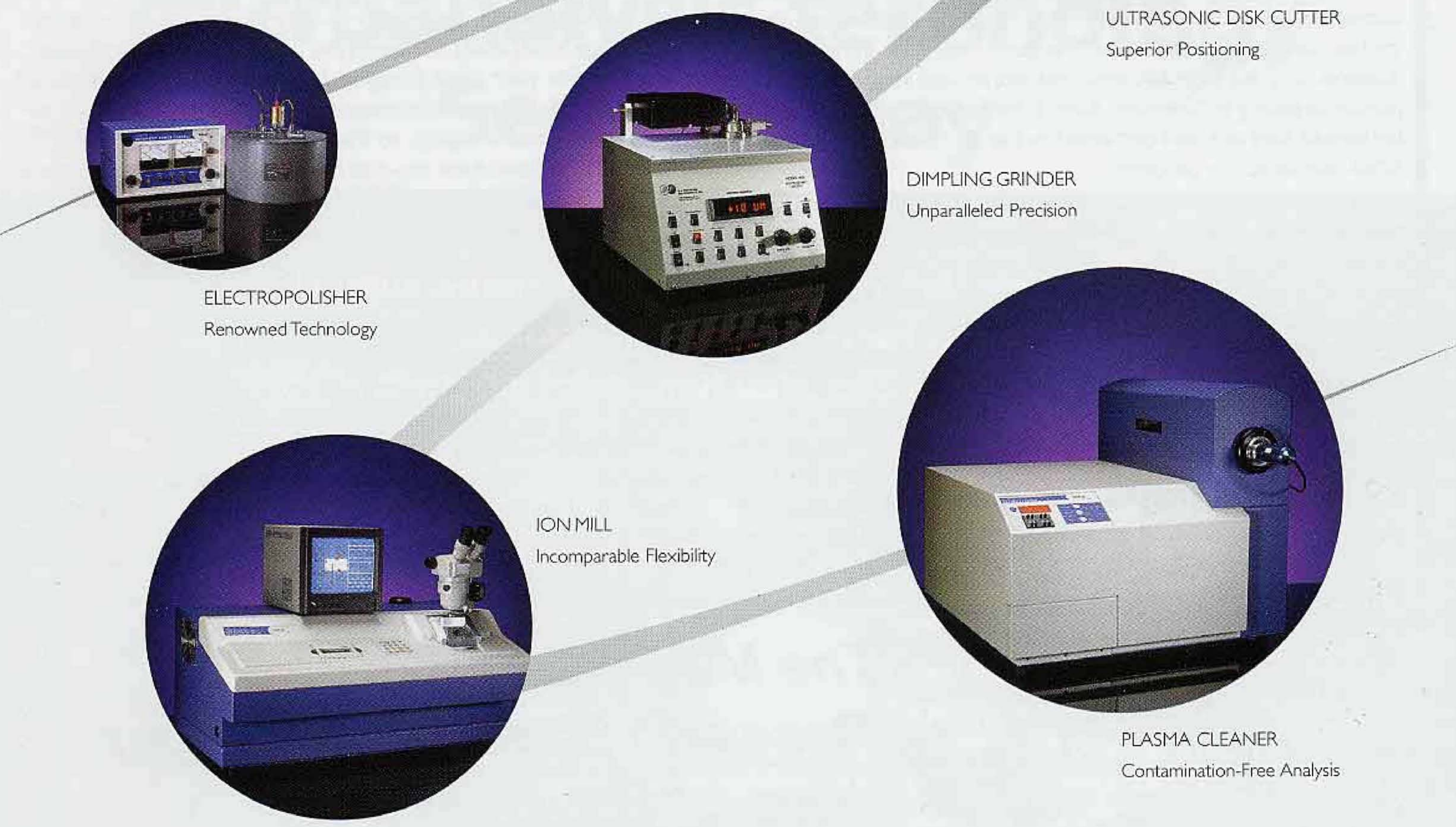

Recognized worldwide for maintaining the highest standards of quality and innovation, Fischione Instruments serves the electron microscopy community by providing state-of-theart instrumentation to meet both existing needs and the requirements of emerging microscopyrelated technologies. 
There are a number of points where stray IR light emits from the cavity and has been known to burn holes through the clothing of an unwitting user (or your friendly customer rep).

While getting the IR beam from the laser cavity to the microscope requires much more user effort than confocal, there are further issues that affect the beam once you get it there. Provided you are using the appropriate IR filters to direct your pulsed IR beam to the objective, both the fluorochrome used and the objective itself can cause poor throughput of your final signal.

Most high NA microscope optics used in confocal are well corrected for visible wavelengths and various aberrations. These visible corrections most often translate into poor infrared transmission. So, the high NA lens that works well for confocal often performs poorly in 2-photon. Most microscope manufacturers offer lenses that are well corrected in the IR these days, but users often overlook this problem.

Fluorochromes are also a cause of much confusion. In theory, the required IR excitation of a particular fluorochrome in 2-photon space is double its visible excitation. This is not set in stone however, and many users have found that standard fluorochromes such as FITC, which has a visible excitation of 485 $\mathrm{nm}$, excites best at $\sim 800 \mathrm{~nm}$ in 2 -photon space. Proper excitation does not necessarily translate into high quantum efficiency, so if a particular fluorochrome does not perform well in 2-photon, it could cause users to increase laser power to maintain an acceptable $\mathrm{S} / \mathrm{N}$ ratio. With most objectives, IR power at the specimen is a few hundred $\mathrm{mW}$ (at $\sim 800 \mathrm{~nm}$ ). This is well above the typical 20 $\mathrm{mW}$ visible lasers used in confocal. Most biological samples will not enjoy this environment, so 2PLSM users must be extremely careful with the attenuation of laser power. All requirements and dangers aside, the maintenance and proper use of these lasers is a far cry from the "hands off" approach to visible tube lasers used in confocal.

The fact that 2PLSM is exponentially more difficult to operate and properly maintain than confocal does not however, cancel its tremendous benefits for some applications routinely utilizing confocal. If your application adheres to the criterion discussed, then 2PLSM may be a welcome enhancement and well worth the effort. If not, stay with confocal or widefield imaging because this technique is most likely not worth the considerable time invested by you and/or your local imaging facility. The small number of criterion listed above encompasses a large number of labs requiring this type of imaging, so there is an undeniable market for 2PLSM. But researchers need to be aware that these machines have specific requirements often restricting this type of imaging to larger multi-user facilities with the technical staff to maintain them.

NOTE

${ }^{\star}$ REES is an abbreviated name for a laser spectrum analyzer made by Rees Instruments Ltd. The REES is a remote optical device coupled to an oscilloscope that allows for real time monitoring of a laser's peak wavelength from $350-1100 \mathrm{~nm}$, and its FWHM (Full Width at Half Maximal) or the laser's bandwidth. The use of a REES greatly simplifies the task of manipulating wavelengths with an IR laser by giving the user a visual readout of precisely where the laser is located in its wavelength range.






\section{INCAFeature}

\section{Visionary \\ microanalysis technology See it today}

Available right now with:

Unique automatic identification of all elements

Simple image assisted set-up

Fast feature detection and data collection algorithm

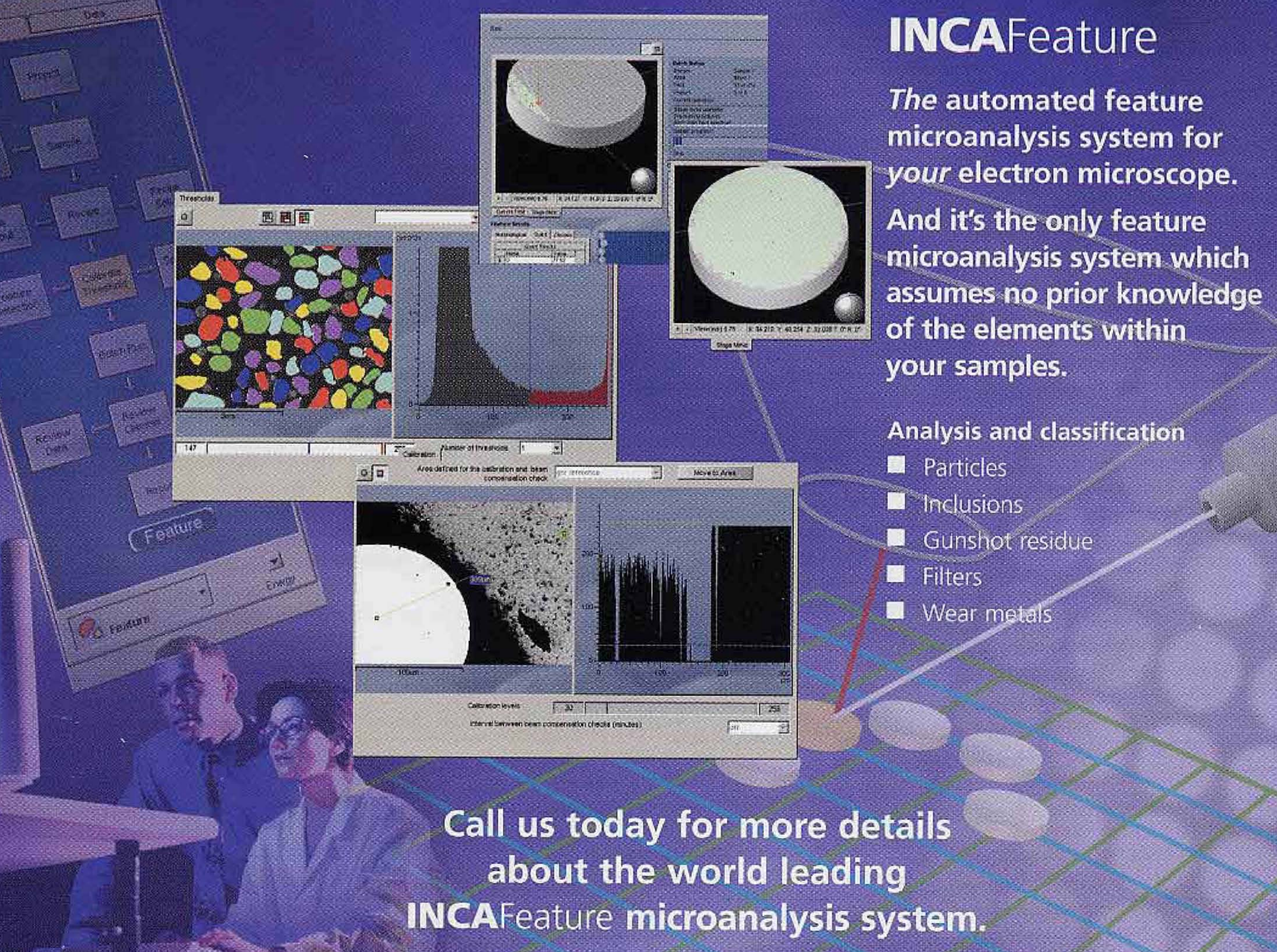

